Supplement of Atmos. Chem. Phys., 21, 5079-5100, 2021

https://doi.org/10.5194/acp-21-5079-2021-supplement

(C) Author(s) 2021. CC BY 4.0 License.

(c) (1)
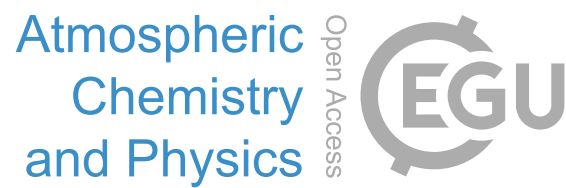

Supplement of

\title{
Reactive organic carbon emissions from volatile chemical products
}

Karl M. Seltzer et al.

Correspondence to: Havala Pye (pye.havala@epa.gov)

The copyright of individual parts of the supplement might differ from the article licence. 
15 Table S1: PUCs, sub-PUCs, NAICS codes, and SCTG codes for all sub-PUCs.

\begin{tabular}{|c|c|c|c|c|}
\hline $\begin{array}{c}\text { Product Use } \\
\text { Categories (PUCs) }\end{array}$ & $\begin{array}{c}\text { Sub-Product Use } \\
\text { Categories (sub-PUCs) }\end{array}$ & NAICS Product Codes ${ }^{\mathrm{a}}$ & $\begin{array}{l}\text { SCTG } \\
\text { Code }^{\text {b }}\end{array}$ & Producer Price Index Category ${ }^{c}$ \\
\hline & Detergents \& Soaps & $\begin{array}{c}3256111,3256114,3256117 \\
325611 \mathrm{~W}\end{array}$ & 233 & $\begin{array}{c}\text { Soap and Other Detergent } \\
\text { Manufacturing }\end{array}$ \\
\hline Cleaning Products & General Cleaners & $\begin{array}{l}3256125,2356127,2356121 \\
235611 \mathrm{~A}, 2356130,325612 \mathrm{~W}\end{array}$ & 233 & $\begin{array}{l}\text { Polish and Other Sanitation Good } \\
\text { Manufacturing; } \\
\text { Soap and Other Detergent } \\
\text { Manufacturing; } \\
\text { Surface Active Agent } \\
\text { Manufacturing; }\end{array}$ \\
\hline & Daily Use Products & $\begin{array}{l}\text { 3256204, 325620D, 325620G, } \\
\text { 325620W, 3256207 (25\%) }\end{array}$ & 232 & Toilet Preparation Manufacturing \\
\hline $\begin{array}{l}\text { Personal care } \\
\text { Products }\end{array}$ & Short Use Products & $\begin{array}{c}\text { 3256201, 325620A, 325611D, } \\
3256207(75 \%)^{\mathbf{d}}\end{array}$ & 232 & $\begin{array}{c}\text { Toilet Preparation Manufacturing; } \\
\text { Soap and Other Detergent } \\
\text { Manufacturing }\end{array}$ \\
\hline Adhesives \& Sealants & Adhesives \& Sealants & $\begin{array}{c}\text { 3255201, 3255204, 3255207, } \\
\text { 305520A, 325520W }\end{array}$ & 239 & Adhesive Manufacturing \\
\hline \multirow{4}{*}{ Paints \& Coatings } & Architectural Coatings & $3255101,325510 \mathrm{~W}$ & $\mathbf{f}$ & Paint and Coating Manufacturing \\
\hline & Aerosol Coatings & $3255107(10 \%)^{\mathbf{e}}$ & $\mathbf{f}$ & Paint and Coating Manufacturing \\
\hline & Allied Paint Products & $325510 \mathrm{~B}$ & $\mathbf{f}$ & Paint and Coating Manufacturing \\
\hline & Industrial Coatings & $\begin{array}{c}3255104, \\
3255107(90 \%)^{\mathrm{e}} \\
\end{array}$ & f & Paint and Coating Manufacturing \\
\hline Printing Inks & Printing Inks & $\begin{array}{l}\text { 3259101, } 3259104,3259107, \\
\text { 325910A, } 325910 \mathrm{E}, 325910 \mathrm{H}, \\
\text { 325910W }\end{array}$ & 231 & Printing Ink Manufacturing \\
\hline \multirow[b]{2}{*}{$\begin{array}{l}\text { Pesticides \& FIFRA } \\
\text { Products }\end{array}$} & FIFRA Pesticides & 3253204,3253207 & 235 & $\begin{array}{l}\text { Pesticide and Other Agricultural } \\
\text { Chemical Manufacturing }\end{array}$ \\
\hline & Agricultural Pesticides & $3251994,3253201,325320 \mathrm{~W}$ & 235 & $\begin{array}{c}\text { All Other Basic Organic Chemical } \\
\text { Manufacturing; Pesticide and Other } \\
\text { Agricultural Chemical } \\
\text { Manufacturing }\end{array}$ \\
\hline Dry Cleaning & Dry Cleaning & \multirow{2}{*}{\multicolumn{3}{|c|}{$\mathrm{g}$}} \\
\hline Oil \& Gas & Oil \& Gas & & & \\
\hline Misc. Products & Misc. Products & \multirow{2}{*}{\multicolumn{3}{|c|}{ h }} \\
\hline Fuels \& Lighter & Fuels \& Lighter & & & \\
\hline
\end{tabular}

a: NAICS Product Codes used for mapping U.S. Census Bureau (2016) ASM statistics to individual sub-PUCs.

b: SCTG Codes used for mapping U.S. Department of Transportation (2015) calculated commodity values to individual sub-PUCs. All values (\$) and mass (tons) quantities retrieved from Table 6 of that report.

c: Category used when retrieving Produce Price Indices from the U.S. Bureau of Labor Statistics, FRED, Federal Reserve Bank of St.

20 Louis.

d: NAICS code 3256207 includes all hair preparation products. Based on sales data by California Air Resources Board 2015 Consumer and Commercial Products Survey Data, we estimate $\sim 75 \%$ of all hair preparation products are short-use (e.g. shampoos, conditioners) and $\sim 25 \%$ are daily-use (e.g. hair spray, other leave-in products).

e: NAICS code 3255107 includes all special-purpose coating materials (e.g. automotive finishing, traffic markings, aerosolized painting

25 products). Based on shipment data from U.S. Census Bureau, Paint and Allied Products - 2010, MA325F(10), Issued July 2011, we estimate $\sim 10 \%$ of all special-purpose coating materials in this NAICS are aerosolized painting products, with the residual consisting of special-purpose coating materials used exclusively in industrial settings.

f: All Paints \& Coatings commodity values retrieved from: U.S. Census Bureau, Paint and Allied Products - 2010, MA325F(10), Issued July 2011.

30 g: sub-PUC usage estimated from solvent usage as reported by The Freedonia Group; Industry Study \#3429; Solvents; July 2016.

h: sub-PUC usage estimated from reported sales data by California Air Resources Board 2015 Consumer and Commercial Products Survey Data: https://ww3.arb.ca.gov/consprod/survey/2015_cp_survey_summary_data_2019-12-09.xlsx; last access: August 28, 2020 
Table S2: PUCs, sub-PUCs, NAICS codes, shipment values, commodity price, producer price index, and product usage for all sub-

PUCs (2016). Per-capita product usage calculated using the U.S. Census Bureau's 2016 estimate of the U.S. population $(\sim 323,000,000)$.

\begin{tabular}{|c|c|c|c|c|c|c|c|c|}
\hline $\begin{array}{l}\text { Product Use } \\
\text { Categories } \\
\text { (PUCs) }\end{array}$ & $\begin{array}{l}\text { Sub-Product Use } \\
\text { Categories (sub- } \\
\text { PUCs) }\end{array}$ & $\begin{array}{l}\text { NAICS } \\
\text { Product } \\
\text { Codes }\end{array}$ & $\begin{array}{c}\text { ASM } \\
\text { Shipment } \\
\text { Values } \\
{[\$ 1000]^{\mathrm{a}}}\end{array}$ & $\begin{array}{l}\text { Commodity } \\
\text { Price }[\$ / k g]^{b}\end{array}$ & $\begin{array}{c}2016 \\
\text { Producer } \\
\text { Price Index }\end{array}$ & $\begin{array}{c}2012 \\
\text { Producer } \\
\text { Price Index }\end{array}$ & $\begin{array}{r}\text { Annu } \\
{[\mathrm{kg} / \text { per }}\end{array}$ & $\begin{array}{l}\text { Jsage } \\
\text { /year] }^{\text {d }}\end{array}$ \\
\hline \multirow{10}{*}{$\begin{array}{l}\text { Cleaning } \\
\text { Products }\end{array}$} & \multirow{4}{*}{$\begin{array}{l}\text { Detergents \& } \\
\text { Soaps }\end{array}$} & 3256111 & 7849011 & \multirow{4}{*}{1.615} & 1.08 & 1.07 & 14.92 & \multirow{4}{*}{40.58} \\
\hline & & 3256114 & 10137793 & & 1.08 & 1.07 & 19.27 & \\
\hline & & 3256117 & 2740950 & & 1.08 & 1.07 & 5.21 & \\
\hline & & $325611 \mathrm{~W}$ & 625339 & & 1.08 & 1.07 & 1.19 & \\
\hline & \multirow{6}{*}{ General Cleaners } & 3256125 & 5391225 & \multirow{6}{*}{1.615} & 1.11 & 1.05 & 9.85 & \multirow{6}{*}{28.47} \\
\hline & & 2356127 & 1024203 & & 1.11 & 1.05 & 1.87 & \\
\hline & & 2356121 & 1067746 & & 1.11 & 1.05 & 1.95 & \\
\hline & & $235611 \mathrm{~A}$ & 290791 & & 1.08 & 1.07 & 0.55 & \\
\hline & & 2356130 & 7173431 & & 1.18 & 1.16 & 13.48 & \\
\hline & & $325612 \mathrm{~W}$ & 417549 & & 1.11 & 1.05 & 0.76 & \\
\hline \multirow{9}{*}{$\begin{array}{l}\text { Personal Care } \\
\text { Products }\end{array}$} & \multirow{5}{*}{$\begin{array}{l}\text { Daily Use } \\
\text { Products }\end{array}$} & 3256204 & 3140488 & \multirow{5}{*}{9.290} & 1.07 & 1.03 & 1.01 & \multirow{5}{*}{8.83} \\
\hline & & $325620 \mathrm{D}$ & 7912985 & & 1.07 & 1.03 & 2.54 & \\
\hline & & $325620 \mathrm{G}$ & 13192677 & & 1.07 & 1.03 & 4.23 & \\
\hline & & $325620 \mathrm{~W}$ & 1562269 & & 1.07 & 1.03 & 0.50 & \\
\hline & & $\begin{array}{c}3256207 \\
(25 \%)^{\mathbf{e}}\end{array}$ & 1734011 & & 1.07 & 1.03 & 0.56 & \\
\hline & \multirow{4}{*}{$\begin{array}{l}\text { Short Use } \\
\text { Products }\end{array}$} & 3256201 & 281282 & \multirow{4}{*}{9.290} & 1.07 & 1.03 & 0.09 & \multirow{4}{*}{3.16} \\
\hline & & $325620 \mathrm{~A}$ & 3574170 & & 1.07 & 1.03 & 1.15 & \\
\hline & & $325611 \mathrm{D}$ & 789937 & & 1.08 & 1.07 & 0.26 & \\
\hline & & $\begin{array}{c}3256207 \\
(75 \%)^{\mathbf{e}}\end{array}$ & 5202032 & & 1.07 & 1.03 & 1.67 & \\
\hline \multirow{5}{*}{$\begin{array}{c}\text { Adhesives \& } \\
\text { Sealants }\end{array}$} & \multirow{5}{*}{$\begin{array}{l}\text { Adhesives \& } \\
\text { Sealants }\end{array}$} & 3255201 & 1405299 & \multirow{5}{*}{2.602} & 1.11 & 1.10 & 1.65 & \multirow{5}{*}{15.23} \\
\hline & & 3255204 & 7967659 & & 1.11 & 1.10 & 9.34 & \\
\hline & & 3255207 & 694706 & & 1.11 & 1.10 & 0.81 & \\
\hline & & $305520 \mathrm{~A}$ & 2405554 & & 1.11 & 1.10 & 2.82 & \\
\hline & & $325520 \mathrm{~W}$ & 513470 & & 1.11 & 1.10 & 0.60 & \\
\hline \multirow{6}{*}{$\begin{array}{l}\text { Paints \& } \\
\text { Coatings }\end{array}$} & \multirow{2}{*}{$\begin{array}{c}\text { Architectural } \\
\text { Coatings }\end{array}$} & 3255101 & 11253306 & \multirow{2}{*}{$2.694^{\mathrm{g}}$} & 1.14 & $1.00^{h}$ & 11.32 & 1227 \\
\hline & & $325510 \mathrm{~W}$ & 1933890 & & 1.14 & $1.00^{\mathrm{h}}$ & 1.95 & 13.21 \\
\hline & Aerosol Coatings & $\begin{array}{c}3255107 \\
(10 \%)^{\mathbf{f}}\end{array}$ & 671641 & $4.616^{\mathrm{g}}$ & 1.14 & $1.00^{\mathrm{h}}$ & 0.39 & 0.39 \\
\hline & $\begin{array}{l}\text { Allied Paint } \\
\text { Products }\end{array}$ & $325510 \mathrm{~B}$ & 1383897 & $2.987^{\mathrm{g}}$ & 1.14 & $1.00^{\mathrm{h}}$ & 1.26 & 1.26 \\
\hline & & 3255104 & 6157635 & $4.310^{\mathrm{g}}$ & 1.14 & $1.00^{\mathrm{h}}$ & 3.87 & \\
\hline & Industrial Coatings & $\begin{array}{c}3255107 \\
(90 \%)^{\mathbf{f}}\end{array}$ & 6044765 & $4.616^{\mathrm{g}}$ & 1.14 & $1.00^{h}$ & 3.55 & 7.42 \\
\hline & & 3259101 & 265917 & & 1.17 & 1.07 & 0.18 & \\
\hline & & 3259104 & 1050099 & & 1.17 & 1.07 & 0.71 & \\
\hline & & 3259107 & 271753 & & 1.17 & 1.07 & 0.18 & \\
\hline Printing Inks & Printing Inks & $325910 \mathrm{~A}$ & 837641 & 4.217 & 1.17 & 1.07 & 0.56 & 3.20 \\
\hline & & $325910 \mathrm{E}$ & 1233022 & & 1.17 & 1.07 & 0.83 & \\
\hline & & $325910 \mathrm{H}$ & 608350 & & 1.17 & 1.07 & 0.41 & \\
\hline & & $325910 \mathrm{~W}$ & 498817 & & 1.17 & 1.07 & 0.34 & \\
\hline & & 3253204 & 905062 & & 1.05 & 1.04 & 0.61 & \\
\hline Pesticides \& & FIFRA Pesticides & 3253207 & 1273582 & 4.552 & 1.05 & 1.04 & 0.86 & 1.46 \\
\hline FIFRA & & 3251994 & 1475958 & & 1.04 & 1.12 & 1.08 & \\
\hline Products & $\begin{array}{l}\text { Agricultural } \\
\text { Pesticides }\end{array}$ & 3253201 & 12842492 & 4.552 & 1.05 & 1.04 & 8.63 & 10.32 \\
\hline & & $325320 \mathrm{~W}$ & 911325 & & 1.05 & 1.04 & 0.61 & \\
\hline Dry Cleaning & Dry Cleaning & & & i & & & & 0.03 \\
\hline Oil \& Gas & Oil \& Gas & & & 1 & & & & 1.32 \\
\hline Misc. Products & Misc. Products & & & & & & & 0.18 \\
\hline $\begin{array}{c}\text { Fuels \& } \\
\text { Lighter }\end{array}$ & Fuels \& Lighter & & & $\mathbf{j}$ & & & & 2.80 \\
\hline
\end{tabular}

${ }^{\text {a}}$ : All values (\$) retrieved via the U.S. Census Bureau ASM's API tool.

b: All quantities retrieved from Table 6 of U.S. Department of Transportation (2015) and representative of 2012 values.

c: U.S. Bureau of Labor Statistics, Producer Price Index by Industry, retrieved from FRED, Federal Reserve Bank of St. Louis. All reindexed to 2010.

40 d: Annual usage $[\mathrm{kg} /$ person/year $]=($ ASM Shipment Values $) \div($ Commodity Price $\times(2016$ Index $/ 2012$ Index $)) \div($ Population $)$ 
e: NAICS code 3256207 includes all hair preparation products. Based on sales data by California Air Resources Board 2015 Consumer and Commercial Products Survey Data, we estimate $75 \%$ of all hair preparation products are short-use (e.g. shampoos, conditioners) and $\sim 25 \%$ are daily-use (e.g. hair spray, other leave-in products).

f: NAICS code 3255107 includes all special-purpose coating materials (e.g. automotive finishing, traffic markings, aerosolized painting

45 products). Based on shipment data from U.S. Census Bureau, Paint and Allied Products - 2010, MA325F(10), Issued July 2011, we

estimate $\sim \mathbf{1 0 \%}$ of all special-purpose coating materials in this NAICS are aerosolized painting products, with the residual consisting of special-purpose coating materials used exclusively in industrial settings.

g: Commodity values retrieved from: U.S. Census Bureau, Paint and Allied Products - 2010, MA325F(10), Issued July 2011.

h: To be consistent with U.S. Census Bureau, Paint and Allied Products - 2010, producer price indices from 2010 used here.

50 i: sub-PUC usage estimated from solvent usage as reported by The Freedonia Group; Industry Study \#3429; Solvents; July 2016.

${ }^{\mathrm{j}}$ : sub-PUC usage estimated from reported sales data by California Air Resources Board 2015 Consumer and Commercial Products Survey Data (CARB, 2019). 
Table S3: Derivation of complete ( $\left(^{\text {st }}\right.$-order and organic) product composition profiles for Adhesives \& Sealants. A similar composite tables was generated for all sub-PUCs,

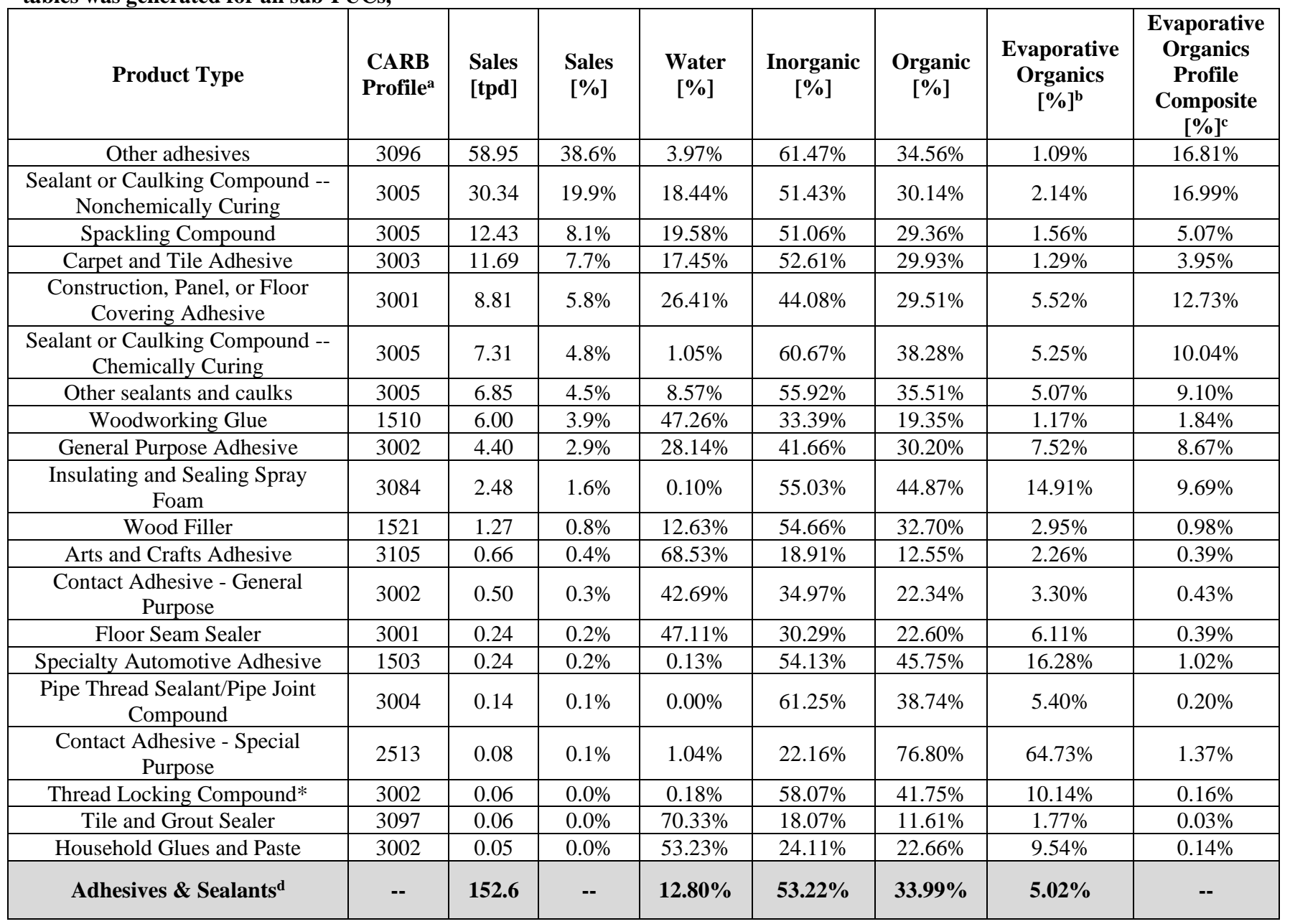

${ }^{\text {a }}$ : Assigned organic profile for each product type. Retrieved from California Air Resources Board and available: https://ww2.arb.ca.gov/speciation-profiles-used-carb-modeling; last access: August 28, 2020

b: "Evaporative Organics" is a component of "Organic." This represents the potentially evaporative organic fraction and excludes "non-evaporative" (i.e. non-volatile) organics, which are not included in the California Air Resource Board's organic profiles.

c: Percent of "Evaporative Organic," weighted by sales abundance:

60 Evaporative Organic Profile Composite [\%]

$$
=(\text { Sales })_{i} \times(\text { Evaporative Organic })_{i} \div\left(\sum_{i=1}^{n}(\text { Sales })_{i} \times(\text { Evaporative Organics })_{i}\right)
$$

Where $i$ is the Product Type index and $\mathbf{n}=20$ (i.e. all Product Types).

d: All water, inorganic, organic, and evaporative organics percentages for the complete sub-PUC are derived on a weighted basis from the reported sales abundance. 
65 Table S4: Organic composition profile source/method summary for all sub-PUCs.

\begin{tabular}{|c|c|c|}
\hline $\begin{array}{l}\text { Product Use Categories } \\
\text { (PUCs) }\end{array}$ & $\begin{array}{l}\text { Sub-Product Use Categories } \\
\text { (sub-PUCs) }\end{array}$ & Organic Composition Source/Methods \\
\hline \multirow{2}{*}{ Cleaning Products } & Detergents \& Soaps & \multirow{5}{*}{$\begin{array}{l}\text { Product type composite derived from CARB's } 2015 \text { Consumer } \\
\text { and Commercial Products Survey }{ }^{\mathbf{b}} \text { and speciated using CARB } \\
\text { organic profiles }\end{array}$} \\
\hline & General Cleaners & \\
\hline \multirow{2}{*}{ Personal Care Products } & Daily Use Products & \\
\hline & Short Use Products & \\
\hline Adhesives \& Sealants & Adhesives \& Sealants & \\
\hline \multirow{4}{*}{ Paints \& Coatings } & Architectural Coatings & $\begin{array}{l}\text { Product type composite derived from CARB's } 2005 \text { Architectural } \\
\text { Coatings Survey }{ }^{\mathbf{d}} \text { (Assumes } 94 \% \text { is water-based, } 6 \% \text { is solvent- } \\
\text { based }^{\mathbf{e}} \text { ) and speciated using CARB organic profiles }{ }^{\mathbf{c}} \text {. }\end{array}$ \\
\hline & Aerosol Coatings & $\begin{array}{l}\text { Product type composite derived from CARB's } 2010 \text { Aerosol } \\
\text { Coatings Survey }{ }^{\mathbf{f}} \text { and speciated using CARB organic profiles }\end{array}$ \\
\hline & Allied Paint Products & $\begin{array}{l}\text { Product type composite derived from CARB's } 2015 \text { Consumer } \\
\text { and Commercial Products Survey }{ }^{\mathbf{b}} \text { and speciated using CARB } \\
\text { organic profiles }\end{array}$ \\
\hline & Industrial Coatings & $\begin{array}{l}\text { SPECIATEv5.0 } \\
\end{array}$ \\
\hline Printing Inks & Printing Inks & SPECIATEv5.0 ${ }^{\mathrm{g}}$ Profile: 2570 \\
\hline \multirow{2}{*}{$\begin{array}{l}\text { Pesticides \& FIFRA } \\
\text { Products }\end{array}$} & FIFRA Pesticides & \multirow{2}{*}{$\begin{array}{c}\text { Product type composite derived from CARB's } 2015 \text { Consumer } \\
\text { and Commercial Products Survey }{ }^{\mathbf{b}} \text { and speciated using CARB } \\
\text { organic profiles }\end{array}$} \\
\hline & Agricultural Pesticides & \\
\hline Dry Cleaning & Dry Cleaning & SPECIATEv5.0 $0^{\mathrm{g}}$ Profile: 2422 \\
\hline Oil \& Gas & Oil \& Gas & a \\
\hline Misc. Products & Misc. Products & \multirow{2}{*}{$\begin{array}{c}\text { Product type composite derived from CARB's } 2015 \text { Consumer } \\
\text { and Commercial Products Surveyb and speciated using CARB } \\
\text { organic profiles }\end{array}$} \\
\hline Fuels \& Lighter & Fuels \& Lighter & \\
\hline
\end{tabular}

${ }^{\text {a}}$ : According to The Freedonia Group; Industry Study \#3429; Solvents; July 2016: 65\% of all solvents used in O\&G operations are alcohols, with the residual largely consisting of "hydrocarbons." We allocate all alcohols to methanol, as it is widely used in and emitted from O\&G operations (Stringfellow, et al., 2017; Lyman et al., 2018; Mansfield et al., 2018). We treat the remaining 35\% as naphtha, a blend of hydrocarbon solvents.

70 b: Ref: CARB, 2019

c: Ref: CARB, 2018

d: Ref: CARB, 2007

e: Ref: CARB, 2014

f: Ref: CARB, 2012

75 g: Ref: EPA, 2019b 
Table S5: Assigned use timescales for all sub-PUCs.

\begin{tabular}{|c|c|c|}
\hline $\begin{array}{c}\text { Product Use Categories } \\
\text { (PUCs) }\end{array}$ & $\begin{array}{c}\text { Sub-Product Use Categories } \\
\text { (sub-PUCs) }\end{array}$ & Use Timescale \\
\hline \multirow{2}{*}{ Cleaning Products } & Detergents \& Soaps & Minutes \\
\cline { 2 - 3 } Personal Care Products & General Cleaners & Days \\
\cline { 2 - 3 } & Daily Use Products & Days \\
\hline Adhesives \& Sealants & Short Use Products & Minutes \\
\hline \multirow{3}{*}{ Paints \& Coatings } & Adhesives \& Sealants & Years \\
\cline { 2 - 3 } & Architectural Coatings & Years \\
\cline { 2 - 3 } & Aerosol Coatings & Years \\
\cline { 2 - 3 } & Allied Paint Products & Years \\
\hline Printing Inks & Industrial Coatings & Years \\
\hline Pesticides \& FIFRA \\
Products & Printing Inks & Years \\
\hline Dry Cleaning & FIFRA Pesticides & Weeks \\
\hline Oil \& Gas & Agricultural Pesticides & Minutes \\
\hline Misc. Products & Dry Cleaning & Years \\
\hline Fuels \& Lighter & Oil \& Gas & Years \\
\hline & Misc. Products & Years \\
\hline
\end{tabular}


Table S6: Methods and Data Sources for Allocating National Emissions to County-level.

\begin{tabular}{|c|c|c|c|}
\hline $\begin{array}{c}\text { Sub-Product Use Categories } \\
\text { (sub-PUCs) }\end{array}$ & $\begin{array}{l}\text { Allocation } \\
\text { Proxy }\end{array}$ & $\begin{array}{c}\text { Employment } \\
\text { NAICS }^{\mathrm{a}}\end{array}$ & NAICS Description \\
\hline Detergents \& Soaps & Population & -- & -- \\
\hline General Cleaners & Population & -- & -- \\
\hline Daily Use Products & Population & -- & -- \\
\hline Short Use Products & Population & -- & -- \\
\hline Adhesives \& Sealants & Population & -- & -- \\
\hline Architectural Coatings & Population & -- & -- \\
\hline Aerosol Coatings & Population & -- & -- \\
\hline Allied Paint Products & Employment & $236 / / /$ & Construction of Buildings \\
\hline \multirow{34}{*}{ Industrial Coatings } & \multirow{34}{*}{ Employment } & 811121 & Automotive Body, Paint, and Interior Repair and Maintenance \\
\hline & & $4411 / /$ & Automobile Dealers \\
\hline & & $4412 / /$ & Other Motor Vehicle Dealers \\
\hline & & 336411 & Aircraft Manufacturing \\
\hline & & $3365 / /$ & Railroad Rolling Stock Manufacturing \\
\hline & & $3366 / /$ & Ship and Boat Building \\
\hline & & 488390 & Other Support Activities for Water Transportation \\
\hline & & $339 / / /$ & Miscellaneous Manufacturing \\
\hline & & $3369 / /$ & Other Transportation Equipment Manufacturing \\
\hline & & $811 / / /$ & Repair and Maintenance \\
\hline & & $3133 / /$ & Textile and Fabric Finishing and Fabric Coating Mills \\
\hline & & 332812 & Metal Coating, Engraving \\
\hline & & $2373 / /$ & Highway, Street, and Bridge Construction \\
\hline & & $321 / / /$ & Wood Product Manufacturing \\
\hline & & 337110 & Wood Kitchen Cabinet and Countertop Manufacturing \\
\hline & & 337121 & Upholstered Household Furniture Manufacturing \\
\hline & & 337122 & Non-upholstered Wood Household Furniture Manufacturing \\
\hline & & 337211 & Wood Office Furniture Manufacturing \\
\hline & & 337212 & Custom Architectural Woodwork and Millwork Manufacturing \\
\hline & & 337124 & Metal Household Furniture Manufacturing \\
\hline & & 337214 & Office Furniture (except Wood) Manufacturing \\
\hline & & 337215 & Showcase, Partition, Shelving, and Locker Manufacturing \\
\hline & & $322 / / /$ & Paper Manufacturing \\
\hline & & 325992 & Photographic Film, Paper, Plate, and Chemical Manufacturing \\
\hline & & $33243 /$ & Metal Can, Box, and Other Metal Container Manufacturing \\
\hline & & $333 / / /$ & Machinery Manufacturing \\
\hline & & $3352 / /$ & Household Appliance Manufacturing \\
\hline & & 331318 & Other Aluminum Rolling, Drawing, and Extruding \\
\hline & & $3314 / /$ & Nonferrous Metal (except Aluminum) Production and Processing \\
\hline & & $33592 /$ & Communication and Energy Wire and Cable Manufacturing \\
\hline & & 335311 & Power, Distribution, and Specialty Transformer Manufacturing \\
\hline & & $3361 / /$ & Motor Vehicle Manufacturing \\
\hline & & $3362 / /$ & Motor Vehicle Body and Trailer Manufacturing \\
\hline & & $3363 / /$ & Motor Vehicle Parts Manufacturing \\
\hline \multirow{2}{*}{ Printing Inks } & \multirow{2}{*}{ Employment } & $32311 /$ & Printing \\
\hline & & $3222 / /$ & Converted Paper Product Manufacturing \\
\hline FIFRA Pesticides & Population & -- & -- \\
\hline Agricultural Pesticides & Pesticide Use & & $\mathbf{b}$ \\
\hline Dry Cleaning & Employment & 812320 & Dry Cleaning and Laundry Services \\
\hline Oil \& Gas & $\begin{array}{l}\text { O\&G Well } \\
\text { Count }\end{array}$ & & c \\
\hline Misc. Products & Population & -- & -- \\
\hline Fuels \& Lighter & Population & -- & -- \\
\hline
\end{tabular}

${ }^{\text {a }}$ All employment mapping, expect Allied Paint Products, follows the NAICS mapping from the 2017 NEI (U.S. EPA, 2017). For Allied

80 Paint Products, mapping is allocated based on construction employment.

b: Allocation of Agriculture Pesticides emissions follows the mapping from the 2017 NEI (U.S. Geological Survey, Pesticide National Synthesis Project, https://water.usgs.gov/nawqa/pnsp/usage/maps/county-level/; last access: August 31, 2020).

c: U.S. Energy Information Administration, The Distribution of U.S. Oil and Natural Gas Wells by Production Rate, Washington, DC, 2019. 
85 Table S7: Observed emission ratios (de Gouw et al., 2017; de Gouw et al., 2018) and inventory emission ratios for Los Angeles County. VCPy: All emissions retrieved from the 2017 NEI, except VCPs, which are replaced using the emissions derived in this study (representative of 2016). 2017 NEI: All emissions retrieved from the 2017 NEI. Emissions consist of all on-road, non-road, non-point, and point sources, as well as biogenic ethanol, methanol, and acetone. Total CO emissions ( 320 Gg) include all on-road, non-road, non-point, and point sources.

\begin{tabular}{|c|c|c|c|}
\hline Compound & $\begin{array}{l}\text { Observed } \\
\text { [g /g CO] }\end{array}$ & $\begin{array}{c}\text { VCPy } \\
\text { [g/g CO] }\end{array}$ & $\begin{array}{l}2017 \text { NEI } \\
\text { [g /g CO] }\end{array}$ \\
\hline Ethanol & 0.0752 & 0.0567 & 0.0301 \\
\hline Acetone & 0.0241 & 0.0265 & 0.0116 \\
\hline i-Propanol & 0.0212 & 0.0134 & 0.0070 \\
\hline Toluene & 0.0112 & 0.0227 & 0.0169 \\
\hline Propane & 0.0211 & 0.0117 & 0.0074 \\
\hline i-Butane & 0.0066 & 0.0079 & 0.0060 \\
\hline$(\mathrm{m}+\mathrm{p})$-Xylenes & 0.0078 & 0.0090 & 0.0041 \\
\hline n-Butane & 0.0103 & 0.0141 & 0.0116 \\
\hline Methyl Ethyl Ketone & 0.0023 & 0.0044 & 0.0017 \\
\hline Methanol & 0.0243 & 0.0187 & 0.0172 \\
\hline Undecane & 0.0018 & 0.0030 & 0.0012 \\
\hline Octanes & 0.0072 & 0.0031 & 0.0014 \\
\hline Heptane & 0.0030 & 0.0026 & 0.0021 \\
\hline Nonane & 0.0015 & 0.0018 & 0.0006 \\
\hline Hexane & 0.0043 & 0.0045 & 0.0039 \\
\hline Methylcyclohexane & 0.0015 & 0.0015 & 0.0007 \\
\hline Trimethylbenzenes & 0.0063 & 0.0032 & 0.0022 \\
\hline Ethyltoluenes & 0.0024 & 0.0030 & 0.0020 \\
\hline Decane & 0.0015 & 0.0012 & 0.0004 \\
\hline Ethylbenzene & 0.0023 & 0.0025 & 0.0019 \\
\hline n-Pentane & 0.0088 & 0.0046 & 0.0043 \\
\hline Dimethylcyclohexanes & 0.0008 & 0.0004 & 0.0001 \\
\hline Styrene & 0.0013 & 0.0013 & 0.0013 \\
\hline Propylbenzenes & 0.0006 & 0.0007 & 0.0004 \\
\hline o-Xylene & 0.0029 & 0.0019 & 0.0018 \\
\hline Methylacetate & 0.0006 & 0.0003 & 0.0001 \\
\hline 2-Methylhexane & 0.0021 & 0.0012 & 0.0011 \\
\hline n-Propanol & 0.0007 & 0.0002 & 0.0001 \\
\hline 3-Methylpentane & 0.0043 & 0.0022 & 0.0022 \\
\hline Cyclohexane & 0.0016 & 0.0018 & 0.0017 \\
\hline
\end{tabular}

90 
Table S8: SCC - SPECIATEv5.0 (EPA, 2019b) profile mapping for all non-point sources in the 2017 NEI. A similar mapping scheme was used to additionally speciate 53 on-road SCCs, 57 non-road SCCs, and $>4,500$ point SCCs.

\begin{tabular}{|c|c|c|c|c|c|c|c|}
\hline SCC & $\begin{array}{c}\begin{array}{c}\text { SPECIATE } \\
\text { Profile }\end{array} \\
\end{array}$ & SCC & $\begin{array}{c}\text { SPECIATE } \\
\text { Profile } \\
\end{array}$ & SCC & $\begin{array}{c}\text { SPECIATE } \\
\text { Profile } \\
\end{array}$ & $\mathrm{SCC}$ & $\begin{array}{c}\text { SPECIATE } \\
\text { Profile }\end{array}$ \\
\hline 2401001000 & 95513 & 2104006000 & 0195 & 2620030000 & 3002 & 2810005001 & 5560 \\
\hline 2401005000 & 2402 & 2104007000 & 0195 & 2102004001 & 0002 & 2810035000 & 5560 \\
\hline 2401008000 & 3135 & 2302002100 & 4553 & 2102004002 & 0002 & 2104002000 & 1185 \\
\hline 2401090000 & 3149 & 2302002200 & 4553 & 2102007000 & 0003 & 2601020000 & 0122 \\
\hline 2401100000 & 3138 & 2302003000 & 4652 & 2304000000 & 1089 & 2103002000 & 1178 \\
\hline 2401200000 & 3138 & 2302003100 & 4651 & 2308000000 & 1008 & 2810005000 & 5560 \\
\hline 2420000000 & 2422 & 2302003200 & 4651 & 2309000000 & 2466 & 2830000000 & 0000 \\
\hline 2425000000 & 1191 & 2501011011 & 8870 & 2312000000 & 0000 & 2505020180 & 2488 \\
\hline 2460100000 & 95509 & 2501011012 & 8870 & 2399000000 & 0000 & 2302070000 & 1188 \\
\hline 2460200000 & 95508 & \begin{tabular}{|l|}
2501011013 \\
\end{tabular} & 8870 & 2510000000 & 0000 & 2862000000 & 0000 \\
\hline 2460400000 & 95510 & 2501011014 & 8870 & 2620000000 & 3002 & 2301000000 & 2462 \\
\hline 2460500000 & 95512 & 2501011015 & 8870 & 2650000000 & 3002 & 2301030000 & 2462 \\
\hline 2460600000 & 95507 & 2501012011 & 8870 & 2660000000 & 8870 & 2635000000 & 8870 \\
\hline 2460800000 & 95511 & 2501012012 & 8870 & 2810030000 & 0000 & 2840000000 & 2402 \\
\hline 2460900000 & 95512 & 2501012013 & 8870 & 2810040000 & 5565 & 2851001000 & 0000 \\
\hline 2461021000 & 1007 & 2501012014 & 8870 & 2810050000 & 0000 & 2501070053 & DIESEVP \\
\hline 2461022000 & 1007 & 2501012015 & 8870 & 2830001000 & 0000 & 2810003000 & 4659 \\
\hline 2461850000 & CARB3103 & 2501060053 & 8870 & 2102001000 & 1185 & 2601000000 & 122 \\
\hline 2415000000 & 8745 & 2501060201 & 8870 & 2102011000 & 0004 & 2601010000 & 122 \\
\hline 2401015000 & 2405 & 2501080050 & 8869 & 2501060052 & 8870 & 2630010000 & 3003 \\
\hline 2401020000 & 2405 & \begin{tabular}{|l|}
2501080100 \\
\end{tabular} & 8869 & 2103004000 & 0002 & 2302070010 & 1188 \\
\hline 2401025000 & 2406 & 2505030120 & 8870 & 2301010000 & 2462 & 2680002000 & 0000 \\
\hline 2401055000 & 3149 & 2610000100 & 0121 & 2302000000 & 4553 & 2306010100 & 0026 \\
\hline 2401075000 & 2414 & 2610000400 & 0121 & 2302050000 & 1188 & 2620010000 & 3002 \\
\hline 2401080000 & 2415 & \begin{tabular}{|l|}
2610000500 \\
\end{tabular} & 0121 & 2302070005 & 1188 & 2102010000 & 0004 \\
\hline 2401070000 & 3131 & 2610030000 & 0121 & 2302080000 & 1188 & 2310010100 & 0003 \\
\hline 2401030000 & 2552 & 2630020000 & 3003 & 2306010000 & 0026 & 2310010200 & 2487 \\
\hline 2401040000 & 2408 & 2680003000 & 8933 & 2505010000 & 2489 & 2310011001 & 1011 \\
\hline 2401060000 & 2411 & 2810025000 & 4553 & 2505020000 & 0305 & 2310011201 & 2487 \\
\hline 2401065000 & 3138 & 2810060100 & 0000 & 2610000300 & 0121 & 2310011501 & 1011 \\
\hline 2401085000 & 2416 & 2810060200 & 0000 & 2640000000 & 0000 & 2310011502 & 1011 \\
\hline 2440000000 & 95512 & 2104004000 & 0002 & 2680001000 & 0000 & 2310011503 & 1011 \\
\hline 2401005700 & 2402 & 2104011000 & 0002 & 2102005000 & 0001 & 2310011505 & 1011 \\
\hline 2401010000 & 3137 & 2501050120 & 8869 & 2302002000 & 4553 & 2310021010 & 2487 \\
\hline 2420000999 & 2422 & 2501055120 & 8869 & 2305000000 & 0000 & 2310021030 & 2487 \\
\hline 2461023000 & 1007 & \begin{tabular}{|l|}
2505040120 \\
\end{tabular} & 8869 & 2307000000 & 2405 & 2310021100 & 0003 \\
\hline 2401050000 & 3127 & \begin{tabular}{|l|}
2102002000 \\
\end{tabular} & 1185 & 2325030000 & 0000 & 2310021300 & 8949 \\
\hline 2420000055 & 0085 & 2102006000 & 0003 & 2501995120 & 8762 & 2310021302 & 1001 \\
\hline 2401045000 & 2409 & 2102008000 & 4642 & 2302070001 & 1188 & 2310021351 & 1001 \\
\hline 2425010000 & 2543 & 2103004001 & 0002 & 2301020000 & 1092 & 2310021400 & 0003 \\
\hline 2425020000 & 2544 & 2103004002 & 0002 & 2801520000 & 3161 & 2310021501 & 8949 \\
\hline 2425030000 & 2545 & \begin{tabular}{|l|}
2103007000 \\
\end{tabular} & 0003 & 2103005000 & 0001 & 2310021502 & 8949 \\
\hline 2425040000 & 1086 & 2103008000 & 4642 & 2103001000 & 1178 & 2310021503 & 8949 \\
\hline 2461020000 & 1007 & 2103011000 & 0002 & 2520010000 & 0000 & 2310021505 & 8949 \\
\hline 2461800001 & CARB3103 & 2302010000 & 4553 & 2501080201 & 8762 & 2310021506 & 8949 \\
\hline 2461800002 & CARB3103 & 2501060051 & 8870 & 2505020030 & 0305 & 2310021603 & 8949 \\
\hline 2440020000 & 95507 & 2102004000 & 0002 & 2505020060 & 0305 & 2310023300 & 8950 \\
\hline 2460000000 & 95512 & 2103006000 & 0003 & 2505020090 & 2488 & 2310023302 & 1001 \\
\hline 2401035000 & 3137 & 2311030000 & 0000 & 2505020120 & 8869 & 2310023351 & 1001 \\
\hline
\end{tabular}




\begin{tabular}{|l|l|}
\hline 2461100000 & 0000 \\
\hline 2310023511 & 8950 \\
\hline 2310023512 & 8950 \\
\hline 2310023513 & 8950 \\
\hline 2310023515 & 8950 \\
\hline 2310023516 & 8950 \\
\hline 2310000220 & 0008 \\
\hline 2310111100 & 1011 \\
\hline 2310111700 & 1011 \\
\hline 2310000660 & 0008 \\
\hline 2310023600 & 8950 \\
\hline 2310121700 & 8949 \\
\hline 2310001000 & 8949 \\
\hline 2310010300 & 8949 \\
\hline 2310021251 & 1001 \\
\hline 2310000553 & 1207 \\
\hline 2310011600 & 1001 \\
\hline 2310021202 & 1001 \\
\hline 2310111401 & 1011 \\
\hline 2310121401 & 8949 \\
\hline 2310121100 & 8949 \\
\hline 2310021303 & 1001 \\
\hline 2310022420 & 8949 \\
\hline 2310002401 & 8949 \\
\hline & \\
\end{tabular}

\begin{tabular}{|c|c|}
\hline 2325000000 & 0000 \\
\hline 2310000552 & 1207 \\
\hline 2310023100 & 0003 \\
\hline 2310023202 & 1001 \\
\hline 2310023251 & 1001 \\
\hline 2310023310 & 8950 \\
\hline 2310023603 & 8950 \\
\hline 2310011500 & 95399 \\
\hline 2310020000 & 8949 \\
\hline 2310022000 & 8949 \\
\hline 2310010700 & DJVNT_R \\
\hline 2310011450 & DJVNT_R \\
\hline 2310021310 & DJVNT_R \\
\hline 2310021509 & DJVNT_R \\
\hline 2310021700 & 1001 \\
\hline 2310030220 & FLR99 \\
\hline 2310030300 & 1207 \\
\hline 2310030400 & 2487 \\
\hline 2310111701 & FLR99 \\
\hline 2310321603 & DJVNT_R \\
\hline 2310400220 & 0008 \\
\hline 2310022010 & $95109 \mathrm{a}$ \\
\hline 2310022090 & 0003 \\
\hline 2310022506 & 1010 \\
\hline
\end{tabular}

\begin{tabular}{|c|c|}
\hline 2505020150 & 100 \\
\hline 2310421100 & 0003 \\
\hline 2310011020 & 2487 \\
\hline 2310021109 & 1001 \\
\hline 2310021209 & 1001 \\
\hline 2310021309 & 1001 \\
\hline 2310021600 & SSJCO_R \\
\hline 2310021602 & SSJCO_R \\
\hline 2310000551 & 1207 \\
\hline 2310023010 & 2487 \\
\hline 2310021102 & 1001 \\
\hline 2310023606 & SSJCB_R \\
\hline 2310023509 & 8950 \\
\hline 2310023102 & 1001 \\
\hline 2310021500 & FLR99 \\
\hline 2310300220 & 0008 \\
\hline 2310321010 & 2487 \\
\hline 2310321100 & 0003 \\
\hline 2310321400 & 0003 \\
\hline 2310421010 & 2487 \\
\hline 2310021802 & 95417 \\
\hline 2310021801 & 95417 \\
\hline 2310021803 & FLR99 \\
\hline
\end{tabular}

\begin{tabular}{|c|c|}
\hline 2310023400 & 0003 \\
\hline 2310421603 & 8949 \\
\hline 2310421400 & 0003 \\
\hline 2310020600 & 1001 \\
\hline 2310011504 & 1011 \\
\hline 2310011506 & 1011 \\
\hline 2310021103 & 1001 \\
\hline 2310021402 & 1001 \\
\hline 2310021403 & 1001 \\
\hline 2310021450 & 8949 \\
\hline 2310021504 & 8949 \\
\hline 2310021101 & 1001 \\
\hline 2310021203 & 1001 \\
\hline 2310021301 & 1001 \\
\hline 2310002000 & 8949 \\
\hline 2310002421 & 8949 \\
\hline 2310012000 & 1011 \\
\hline 2310012020 & $95087 \mathrm{a}$ \\
\hline 2310012526 & 1011 \\
\hline 2310022105 & 0008 \\
\hline 2310112401 & 1011 \\
\hline 2310011100 & 0003 \\
\hline 2310000230 & 0008 \\
\hline
\end{tabular}


Table S9: National-level emission rates [ $\mathrm{kg} \mathrm{person}^{-1}$ year $^{-1}$ ] for the top-200 compounds emitted from VCPs, as predicted by VCPy.

\begin{tabular}{|c|c|c|c|c|c|c|c|}
\hline Compound & Emissions & Compound & Emissions & Compound & Emissions & Compound & Emissions \\
\hline Ethanol & 1.6519 & C6 Cycloalkanes & 0.0238 & C10 Trialkylbenzenes & 0.0052 & \begin{tabular}{|c|} 
cis- 1, cis-3,5- \\
trimethylcyclohexane \\
\end{tabular} & 0.0022 \\
\hline Acetone & 0.8506 & d-Limonene & 0.0235 & Aliphatics & 0.0052 & \begin{tabular}{|c|} 
trans,cis-1,2,4- \\
trimethylcyclohexane \\
\end{tabular} & 0.0022 \\
\hline Isopropyl Alcohol & 0.4274 & C15 Cycloalkanes & 0.0223 & Isobutyl Acetate & 0.0051 & $\begin{array}{c}1,1,3- \\
\text { trimethylcyclohexane }\end{array}$ & 0.0022 \\
\hline Toluene & 0.3704 & \begin{tabular}{|c|} 
Other, Misc. VOC \\
Compounds \\
Aggregated In Profile
\end{tabular} & 0.0219 & $\begin{array}{c}\text { C11 Tetrasubstituted } \\
\text { Benzenes }\end{array}$ & 0.0051 & $\begin{array}{c}1,1,3- \\
\text { trimethylcyclopentane }\end{array}$ & 0.0022 \\
\hline n-Tetradecane & 0.3632 & $\begin{array}{l}\text { Branched C10 } \\
\text { Alkanes }\end{array}$ & 0.0208 & 2,6-dimethylnonane & 0.0050 & 4-methyldecane & 0.0022 \\
\hline Fragrances & 0.3444 & C10 Cycloalkanes & 0.0208 & Methyl Acetate & 0.0050 & $\begin{array}{c}1,2,3- \\
\text { Trimethylbenzene }\end{array}$ & 0.0021 \\
\hline Propane & 0.3365 & Witch Hazel & 0.0197 & 3-methylheptane & 0.0049 & 5-methyldecane & 0.0021 \\
\hline $\begin{array}{l}\text { Volatile Methyl } \\
\text { Siloxanes } \\
\end{array}$ & 0.3024 & \begin{tabular}{|c|} 
2-Amino-2-Methyl-1- \\
Propanol
\end{tabular} & 0.0188 & 2-methylhexane & 0.0049 & \begin{tabular}{|c|} 
trans,trans-1,3,5- \\
trimethylcyclohexane \\
\end{tabular} & 0.0021 \\
\hline Isobutane & 0.2814 & n-Tridecane & 0.0186 & 1-Tetradecene & 0.0049 & Butylcyclohexane & 0.0021 \\
\hline Propylene Glycol & 0.2488 & n-Heptane & 0.0167 & C15 Branched Alkanes & 0.0048 & 4-methylheptane & 0.0021 \\
\hline $\begin{array}{c}\text { 2,2,4-Trimethyl-1,3- } \\
\text { Pentanediol } \\
\text { Isobutyrate (Texanol) } \\
\end{array}$ & 0.2195 & Hexane & 0.0162 & \begin{tabular}{|c|} 
Other, Lumped VOCs, \\
Individually $<2 \%$ Of \\
Category
\end{tabular} & 0.0047 & 2,3-Dimethylbutane & 0.0021 \\
\hline Ethylene Glycol & 0.2144 & $\begin{array}{l}\text { Propylene Glycol } \\
\text { Monomethyl Ether } \\
\text { Acetate }\end{array}$ & 0.0159 & $\begin{array}{c}1,2,4- \\
\text { trimethylcyclopentane }\end{array}$ & 0.0046 & C11 Tetralin or Indane & 0.0020 \\
\hline Xylenes & 0.1941 & Benzene & 0.0155 & \begin{tabular}{|c|}
$1,2-$ \\
dimethylcyclopentane
\end{tabular} & 0.0046 & Trichloroethylene & 0.0020 \\
\hline n-Butane & 0.1916 & C7 Cycloalkanes & 0.0151 & Turpentine & 0.0046 & Diisopropyl Adipate & 0.0020 \\
\hline Methanol & 0.1772 & C14 Cycloalkanes & 0.0150 & Diethylene Glycol & 0.0045 & $\begin{array}{l}\text { 1,3-diethylbenzene } \\
\text { (meta) }\end{array}$ & 0.0019 \\
\hline $\begin{array}{l}\text { Ethylene Glycol } \\
\text { Monobutyl Ether }\end{array}$ & 0.1588 & n-Pentane & 0.0149 & C12 Naphthalenes & 0.0045 & 2,2-Dimethylbutane & 0.0018 \\
\hline $\begin{array}{c}\text { Branched C12 } \\
\text { Alkanes }\end{array}$ & 0.1568 & Branched C7 Alkanes & 0.0146 & \begin{tabular}{|c|} 
Propylene Glycol \\
Monomethyl Ether (1- \\
Methoxy-2-propanol) \\
\end{tabular} & 0.0045 & Dihydroxyacetone & 0.0018 \\
\hline n-Undecane & 0.1461 & Ethyl Cyanoacrylate & 0.0139 & n-Propyl Alcohol & 0.0044 & Dipropylene Glycol & 0.0017 \\
\hline $\begin{array}{c}\text { Methylene Chloride } \\
\text { (Dichloromethane) }\end{array}$ & 0.1291 & C13 Cycloalkanes & 0.0129 & \begin{tabular}{|c|} 
trans,trans-1,2,4- \\
trimethylcyclohexane
\end{tabular} & 0.0043 & Dimethyl Succinate & 0.0016 \\
\hline $\begin{array}{c}\text { Methyl Ethyl Ketone } \\
\text { (2-Butanone) }\end{array}$ & 0.1287 & Isopropyl acetate & 0.0126 & $\begin{array}{l}\text { Diethylene Glycol } \\
\text { Monoethyl Ether }\end{array}$ & 0.0043 & $\begin{array}{l}\text { Ethylene Glycol } \\
\text { Monopropyl Ether }\end{array}$ & 0.0015 \\
\hline Dimethyl Ether & 0.1186 & \begin{tabular}{|c|}
$\begin{array}{c}\text { Propylene Glycol N- } \\
\text { Propyl Ether }\end{array}$ \\
\end{tabular} & 0.0124 & C5 Branched Alkanes & 0.0042 & Triethanolamine & 0.0015 \\
\hline n-Dodecane & 0.1100 & $\begin{array}{c}1,1,1,2- \\
\text { Tetrafluoroethane } \\
\text { (HFC-134a) }\end{array}$ & 0.0124 & Hexadecane & 0.0040 & p-Xylene & 0.0015 \\
\hline $\begin{array}{c}\text { 1,1-Difluoroethane } \\
\text { (HFC-152a) }\end{array}$ & 0.1082 & $\begin{array}{c}\text { Hydrocarbon } \\
\text { Propellant (LPG) }\end{array}$ & 0.0122 & m-Xylene & 0.0040 & C11 Dialkyl Benzenes & 0.0014 \\
\hline $\begin{array}{c}\text { Hydrocarbon } \\
\text { Propellant (LPG, } \\
\text { Sweetened) } \\
\end{array}$ & 0.0914 & Cyclopentane & 0.0118 & Branched C17 Alkanes & 0.0040 & C14 Branched Alkanes & 0.0014 \\
\hline C11 Cycloalkanes & 0.0898 & $\begin{array}{l}\text { N,N-Diethyl-M- } \\
\text { Toluamide }\end{array}$ & 0.0115 & Methyl Amyl Ketone & 0.0040 & $\begin{array}{l}\text { Methyl Ethyl } \\
\text { Ketoxime }\end{array}$ & 0.0014 \\
\hline n-Octane & 0.0803 & Styrene & 0.0112 & $\begin{array}{c}1,2,4- \\
\text { Trimethylbenzene } \\
\end{array}$ & 0.0038 & $\begin{array}{c}2,2,4,6,6- \\
\text { Pentamethylheptane } \\
\end{array}$ & 0.0014 \\
\hline C12 Cycloalkanes & 0.0758 & C16 Cycloalkanes & 0.0109 & $\begin{array}{l}\text { Misc. Oxygenated } \\
\text { Compounds }\end{array}$ & 0.0037 & \begin{tabular}{|c|} 
(2- \\
methylpropyl)benzene \\
(or isobutylbenzene)
\end{tabular} & 0.0014 \\
\hline $\begin{array}{c}\text { C13 Branched } \\
\text { Alkanes }\end{array}$ & 0.0747 & $\begin{array}{c}1,3,5- \\
\text { trimethylbenzene }\end{array}$ & 0.0107 & C10 Dialkyl Benzenes & 0.0035 & Toluene & 0.0013 \\
\hline $\begin{array}{c}\text { Branched C9 } \\
\text { Alkanes } \\
\end{array}$ & 0.0700 & \begin{tabular}{|c|}
$\mathrm{N}-$ \\
Methylpyrrolidinone \\
\end{tabular} & 0.0107 & $\begin{array}{l}\text { Dipropylene Glycol } \\
\text { Monopropyl Ether }\end{array}$ & 0.0034 & $\begin{array}{c}\text { Methyl Propyl Ketone } \\
\text { (2-Pentanone) }\end{array}$ & 0.0013 \\
\hline
\end{tabular}




\begin{tabular}{|c|c|c|c|c|c|c|c|}
\hline C8 Cycloalkanes & 0.0617 & $\begin{array}{c}\text { Aggregated Vocs < } \\
1.0 \%\end{array}$ & 0.0104 & Phenoxyethanol & 0.0033 & Methyltriacetoxysilane & 0.0013 \\
\hline Pine Oil & 0.0605 & $\begin{array}{c}\text { C11 Trialkyl } \\
\text { Benzenes } \\
\end{array}$ & 0.0101 & Diethanolamine & 0.0033 & Ethyltriacetoxysilane & 0.0012 \\
\hline n-Nonane & 0.0551 & Benzyl Alcohol & 0.0099 & m-Xylene & 0.0032 & $\begin{array}{c}\text { Hexylene Glycol (2- } \\
\text { Methyl-2,4- } \\
\text { Pentanediol) }\end{array}$ & 0.0012 \\
\hline $\begin{array}{l}\text { Branched C11 } \\
\text { alkanes }\end{array}$ & 0.0538 & $\begin{array}{c}\text { C12 Trisubstituted } \\
\text { Benzenes }\end{array}$ & 0.0092 & Diisobutyl Ketone & 0.0031 & Isobutyl Alcohol & 0.0012 \\
\hline Ethyl Acetate & 0.0481 & \begin{tabular}{|c|} 
cis-1,3- \\
dimethylcyclohexane \\
\end{tabular} & 0.0090 & 3-Methylpentane & 0.0030 & Ethylbenzene & 0.0012 \\
\hline $\begin{array}{l}\text { Perchloroethylene } \\
\text { (Tetrachloroethene) }\end{array}$ & 0.0481 & White Mineral Oil & 0.0089 & Methyl Methacrylate & 0.0030 & $\begin{array}{l}\text { 1,2-diethylbenzene } \\
\text { (ortho) }\end{array}$ & 0.0012 \\
\hline C9 Cycloalkanes & 0.0474 & $\begin{array}{l}\text { Diethylene Glycol } \\
\text { Monomethyl Ether }\end{array}$ & 0.0086 & $\begin{array}{l}\text { 1-Ethyl-2-Propyl } \\
\text { Cyclohexane }\end{array}$ & 0.0029 & Diacetone Alcohol & 0.0012 \\
\hline n-Decane & 0.0445 & o-Xylene & 0.0085 & Dimethyl Adipate & 0.0028 & Tetramethylbenzenes & 0.0012 \\
\hline n-Heptane & 0.0422 & n-Pentadecane & 0.0083 & Ethylcyclohexane & 0.0028 & $\begin{array}{c}1,3,5- \\
\text { Trimethylbenzene }\end{array}$ & 0.0011 \\
\hline $\begin{array}{c}\text { Branched C8 } \\
\text { Alkanes } \\
\end{array}$ & 0.0407 & 2-Methylheptane & 0.0076 & \begin{tabular}{|c|} 
trans-1,4- \\
dimethylcyclohexane \\
\end{tabular} & 0.0027 & $\begin{array}{l}\text { Misc. Hydrocarbon } \\
\text { Propellants } \\
\end{array}$ & 0.0011 \\
\hline Ethanolamine & 0.0381 & $\begin{array}{l}\text { C16 Branched } \\
\text { Alkanes }\end{array}$ & 0.0072 & Cumene & 0.0027 & $\begin{array}{c}\text { 1,4-diethylbenzene } \\
\text { (para) }\end{array}$ & 0.0010 \\
\hline Methylcyclohexane & 0.0369 & o-Ethyltoluene & 0.0072 & $\begin{array}{c}\text { trans-1,3- } \\
\text { dimethylcyclohexane } \\
\end{array}$ & 0.0027 & Diisopropylene glycol & 0.0010 \\
\hline $\begin{array}{l}\text { Dipropylene Glycol } \\
\text { Monomethyl Ether }\end{array}$ & 0.0307 & 2-Methylpentane & 0.0071 & 4-methylnonane & 0.0026 & C13 Naphthalenes & 0.0010 \\
\hline $\begin{array}{c}\text { Branched C6 } \\
\text { Alkanes }\end{array}$ & 0.0306 & $\begin{array}{c}\text { Voc Ingredients < } \\
0.1 \%\end{array}$ & 0.0068 & Cyclohexane & 0.0026 & $\begin{array}{l}\text { Ethylene Glycol } \\
\text { Monohexyl Ether }\end{array}$ & 0.0010 \\
\hline n-Hexane & 0.0302 & Glycerol & 0.0067 & 2-methyldecane & 0.0024 & Tetrahydrofuran & 0.0010 \\
\hline $\begin{array}{l}\text { Diethylene Glycol } \\
\text { Monobutyl Ether }\end{array}$ & 0.0297 & n-Propylbenzene & 0.0065 & 2-Ethylhexyl Benzoate & 0.0024 & Cyclohexanol & 0.0010 \\
\hline Ethyl Benzene & 0.0286 & $\begin{array}{c}\text { Propylene Glycol } \\
\text { Butyl Ether (1- } \\
\text { Butoxy-2-Propanol) } \\
\end{array}$ & 0.0063 & $\begin{array}{l}\text { trans 1-methyl-3- } \\
\text { propyl cyclohexane }\end{array}$ & 0.0024 & Methylindans & 0.0010 \\
\hline m-Ethyltoluene & 0.0281 & $\begin{array}{c}1,2,3- \\
\text { trimethylbenzene }\end{array}$ & 0.0059 & 3-methyldecane & 0.0023 & Diethyl Phthalate & 0.0010 \\
\hline $\begin{array}{c}1,2,4- \\
\text { Trimethylbenzene }\end{array}$ & 0.0270 & $\begin{array}{c}\text { Ethyl-3- } \\
\text { Ethoxypropionate }\end{array}$ & 0.0056 & 2,6-dimethylheptane & 0.0023 & Isopentane & 0.0010 \\
\hline N-Butyl Acetate & 0.0253 & \begin{tabular}{|c|} 
Glycol Ether Dpnb (1- \\
(2-Butoxy-1- \\
Methylethoxy)-2- \\
Propanol) \\
\end{tabular} & 0.0056 & Misc. Esters & 0.0023 & Triethylene Glycol & 0.0009 \\
\hline $\begin{array}{l}\text { Methyl Isobutyl } \\
\text { Ketone (Hexone) }\end{array}$ & 0.0248 & n-Butyl Alcohol & 0.0055 & Butyl Acrylate & 0.0022 & $\begin{array}{l}\text { Pentanedioic Acid, } \\
\text { Dimethyl Ester }\end{array}$ & 0.0009 \\
\hline
\end{tabular}


Table S10: Tabulation of Fig. 4 from main text.

\begin{tabular}{|c|c|c|c|c|c|}
\hline $\begin{array}{c}\text { Product Use Categories } \\
\text { (PUCs) }\end{array}$ & $\mathbf{2 . 5}^{\text {th }}$ & $\mathbf{2 5}^{\text {th }}$ & Mean & $\mathbf{7 5}^{\text {th }}$ & $\mathbf{9 7 . 5 t h ~}^{\text {th }}$ \\
\hline Cleaning Products & 1.25 & 1.72 & 2.02 & 2.3 & 2.94 \\
\hline Personal Care Products & 1.32 & 1.76 & 2.01 & 2.25 & 2.81 \\
\hline Adhesives \& Sealants & 0.51 & 0.66 & 0.76 & 0.85 & 1.05 \\
\hline Paints \& Coatings & 2.53 & 2.92 & 3.12 & 3.34 & 3.77 \\
\hline Printing Inks & 0.54 & 0.7 & 0.8 & 0.89 & 1.1 \\
\hline Pesticides \& FIFRA Products & 0.40 & 0.52 & 0.58 & 0.65 & 0.78 \\
\hline Other & 0.02 & 0.06 & 0.16 & 0.24 & 0.46 \\
\hline Total & 8.07 & 8.98 & 9.46 & 9.96 & 10.94 \\
\hline
\end{tabular}

100 
Table S11: Mapping of all sub-PUCs to equivalent Source Classification Codes (SCCs).

\begin{tabular}{|c|c|c|c|}
\hline \multirow{2}{*}{ PUC } & \multirow{2}{*}{$\mathrm{SCC}$} & \multirow{2}{*}{ SCC Description } & VCPy \\
\hline & & & sub-PUC \\
\hline \multirow{3}{*}{ Cleaning Products } & 2460200000 & All Household Products & \multirow{3}{*}{$\begin{array}{c}\text { Detergents \& Soaps } \\
\text { General Cleaners }\end{array}$} \\
\hline & 2415000000 & Degreasing & \\
\hline & 2460400000 & All Automotive Aftermarket Products & \\
\hline \multirow{2}{*}{ Personal Care Products } & \multirow{2}{*}{2460100000} & \multirow{2}{*}{ All Personal Care Products } & Daily Use Products \\
\hline & & & Short Use Products \\
\hline Adhesives \& Sealants & 2460600000 & All Adhesives and Sealants & Adhesives and Sealants \\
\hline \multirow{20}{*}{ Paints \& Coatings } & 2401001000 & Architectural Coatings & Architectural Coatings \\
\hline & 2460500000 & All Coatings and Related Products & Aerosol Coatings \\
\hline & 2401005000 & Auto Refinishing & \multirow{17}{*}{ Industrial Coatings } \\
\hline & 2401008000 & Traffic Markings & \\
\hline & 2401015000 & Factory Finished Wood & \\
\hline & 2401020000 & Wood Furniture & \\
\hline & 2401025000 & Metal Furniture & \\
\hline & 2401030000 & Paper & \\
\hline & 2401040000 & Metal Cans & \\
\hline & 2401055000 & Machinery and Equipment & \\
\hline & 2401060000 & Large Appliances & \\
\hline & 2401065000 & Electronic and Other Electrical & \\
\hline & 2401070000 & Motor Vehicles & \\
\hline & 2401075000 & Aircraft & \\
\hline & 2401085000 & Railroad & \\
\hline & 2401080000 & Marine & \\
\hline & 2401090000 & Misc. Manufacturing & \\
\hline & 2401100000 & Industrial Maintenance Coatings & \\
\hline & 2401200000 & Other Special Purpose Coatings & \\
\hline & 2402000000 & Paint Strippers & Allied Paint Products \\
\hline Printing Inks & 2425000000 & Graphic Arts, employment & Printing Inks \\
\hline \multirow{2}{*}{ Pesticides \& FIFRA Products } & 2460800000 & All FIFRA Related Products & FIFRA Pesticides \\
\hline & 2461850000 & Pesticide Application: Agricultural & Agricultural Pesticides \\
\hline Dry Cleaning & 2420000000 & Dry Cleaning & Dry Cleaning \\
\hline Oil \& Gas & $\mathrm{n} / \mathrm{a}$ & $\mathrm{n} / \mathrm{a}$ & Oil \& Gas \\
\hline Misc. Products & 2460900000 & Miscellaneous Products: NEC & Misc. Products \\
\hline Fuels and Lighter & $\mathrm{n} / \mathrm{a}$ & $\mathrm{n} / \mathrm{a}$ & Fuels and Lighter \\
\hline
\end{tabular}




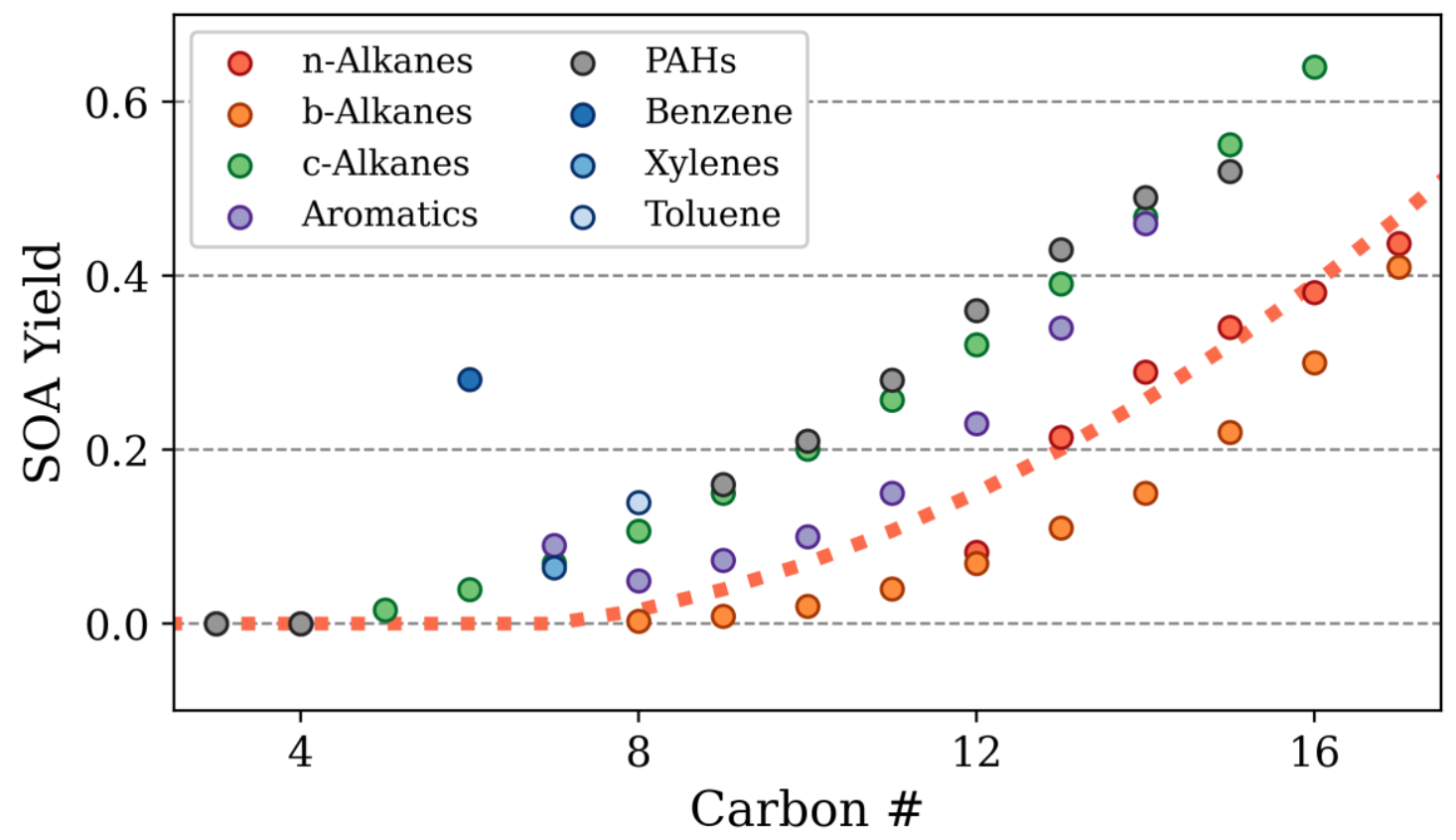

105 Figure S1: Summary of SOA yields by compound class. Several references are used to construct these values and are described in the main text. 


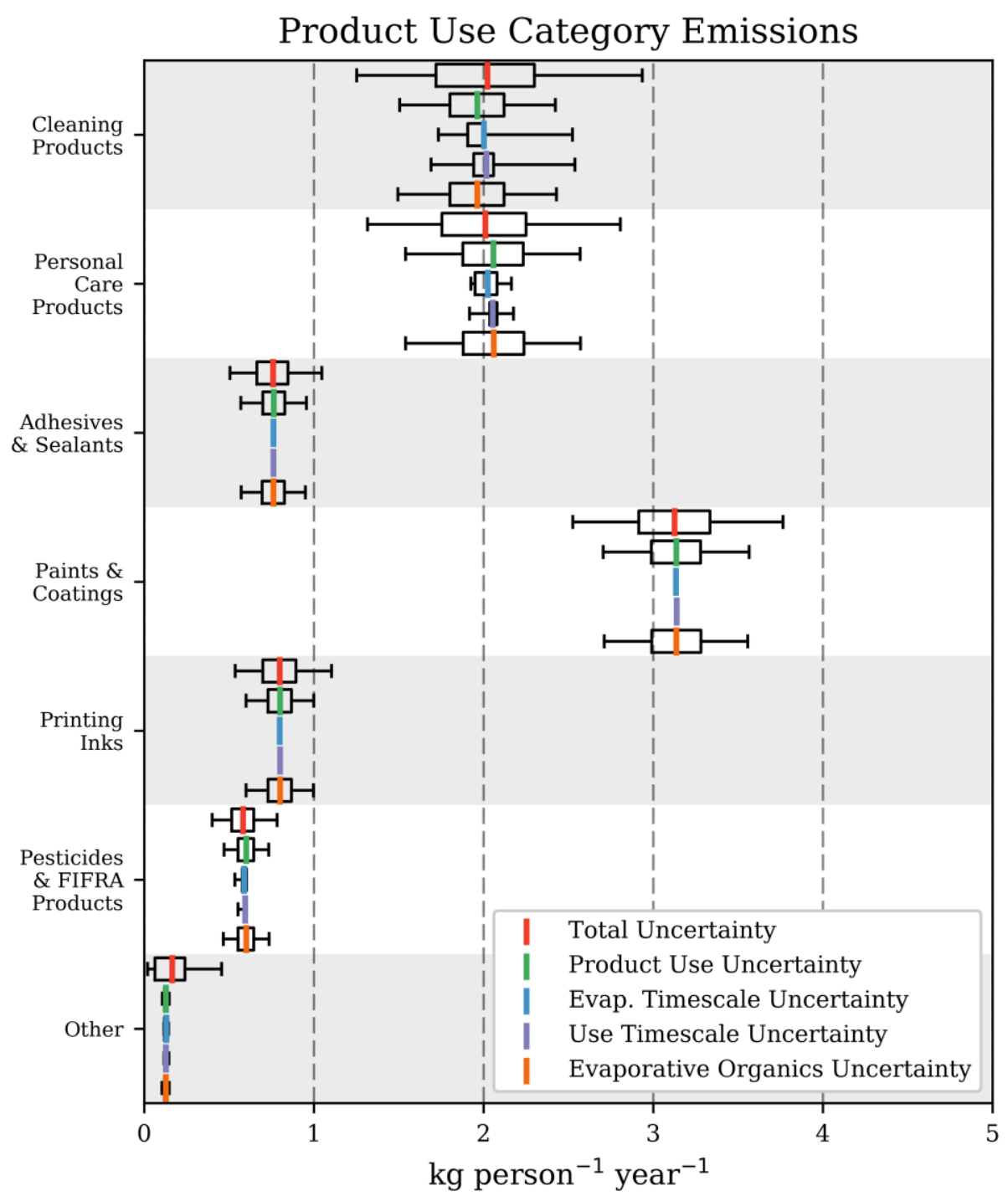

Figure S2: Monte Carlo sensitivity results: mean, interquartile range, and 95\% confidence intervals for emission rates for six major PUCs and the sum of all others. Red: Estimate considering uncertainty in product usage, evaporation timescale, use timescale, and controls. Green: MC simulations that only perturb product usage uncertainties. Blue: MC simulations that only perturb evaporation timescale uncertainties. Purple: MC simulations that only perturb use timescale uncertainties. Orange: MC simulations that only perturb evaporative organic uncertainties. 

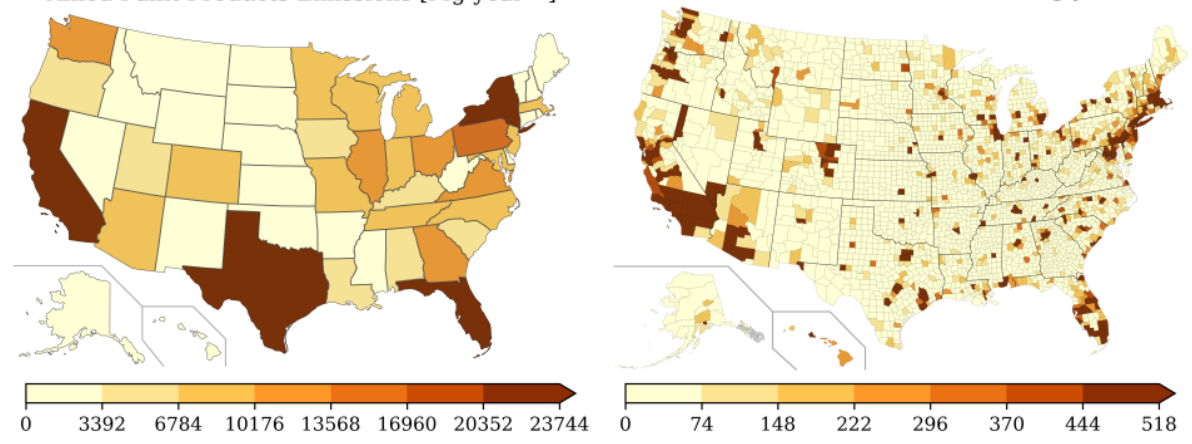
Industrial Coatings Emissions [Mg year ${ }^{-1}$ ]

Industrial Coatings Emissions [ $\mathrm{Mg}$ year $^{-1}$ ]
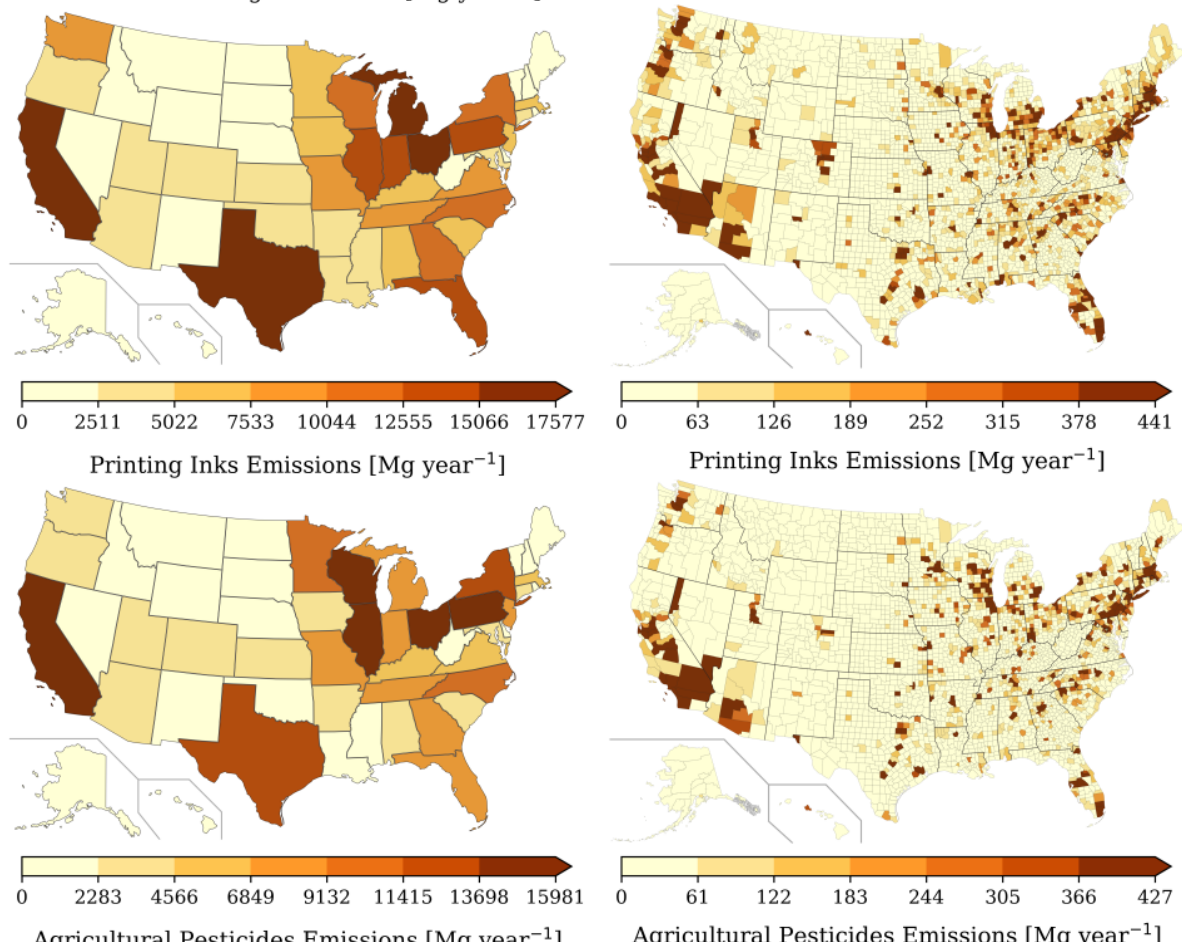

Agricultural Pesticides Emissions [Mg year ${ }^{-1}$ ]

Agricultural Pesticides Emissions [Mg year ${ }^{-1}$ ]
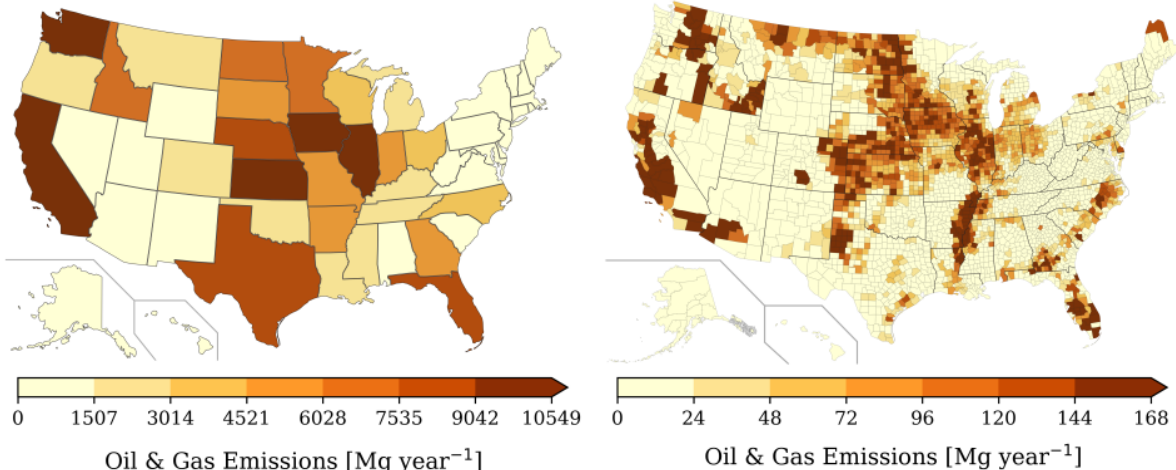

Oil \& Gas Emissions [Mg year ${ }^{-1}$ ]
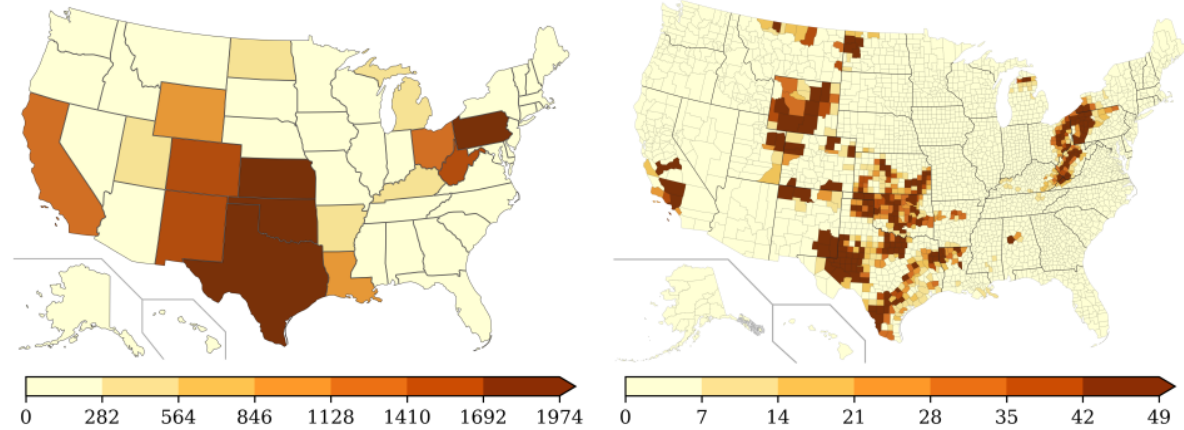

Figure S3: State- and County-level emissions for select sub-PUCs. 


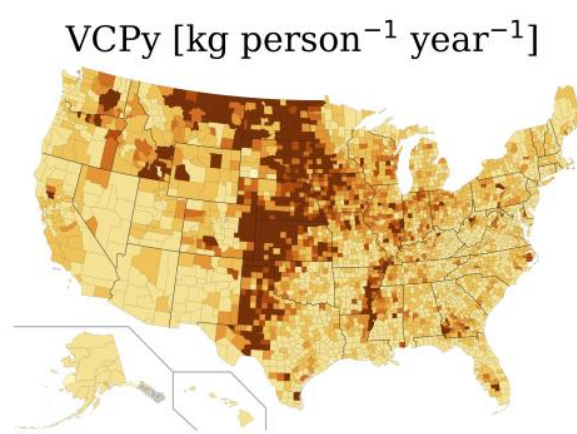

(a)

120

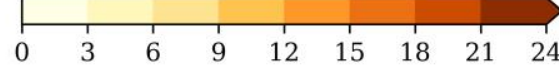

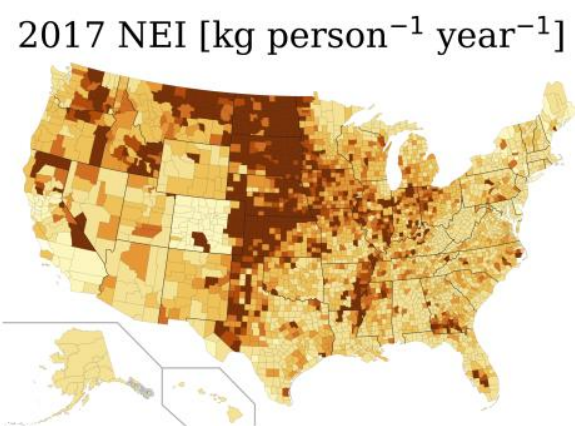

(b)
VCPy / 2017 NEI Ratio

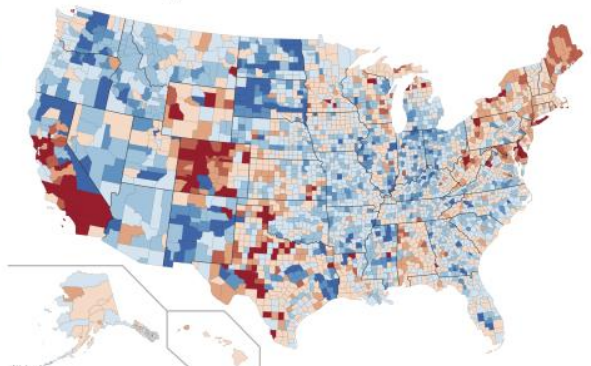

(c)

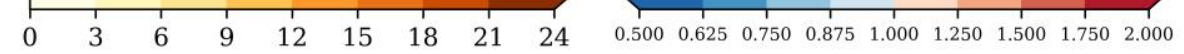

Figure S4: (a) County-level per-capita VCP emissions from the VCPy inventory (same as right panel of Fig. 5 in main text), (b) County-level per-capita VCP emissions from the 2017 NEI, and (c) County-level ratio of VCPy / 2017 NEI VCP emissions. 


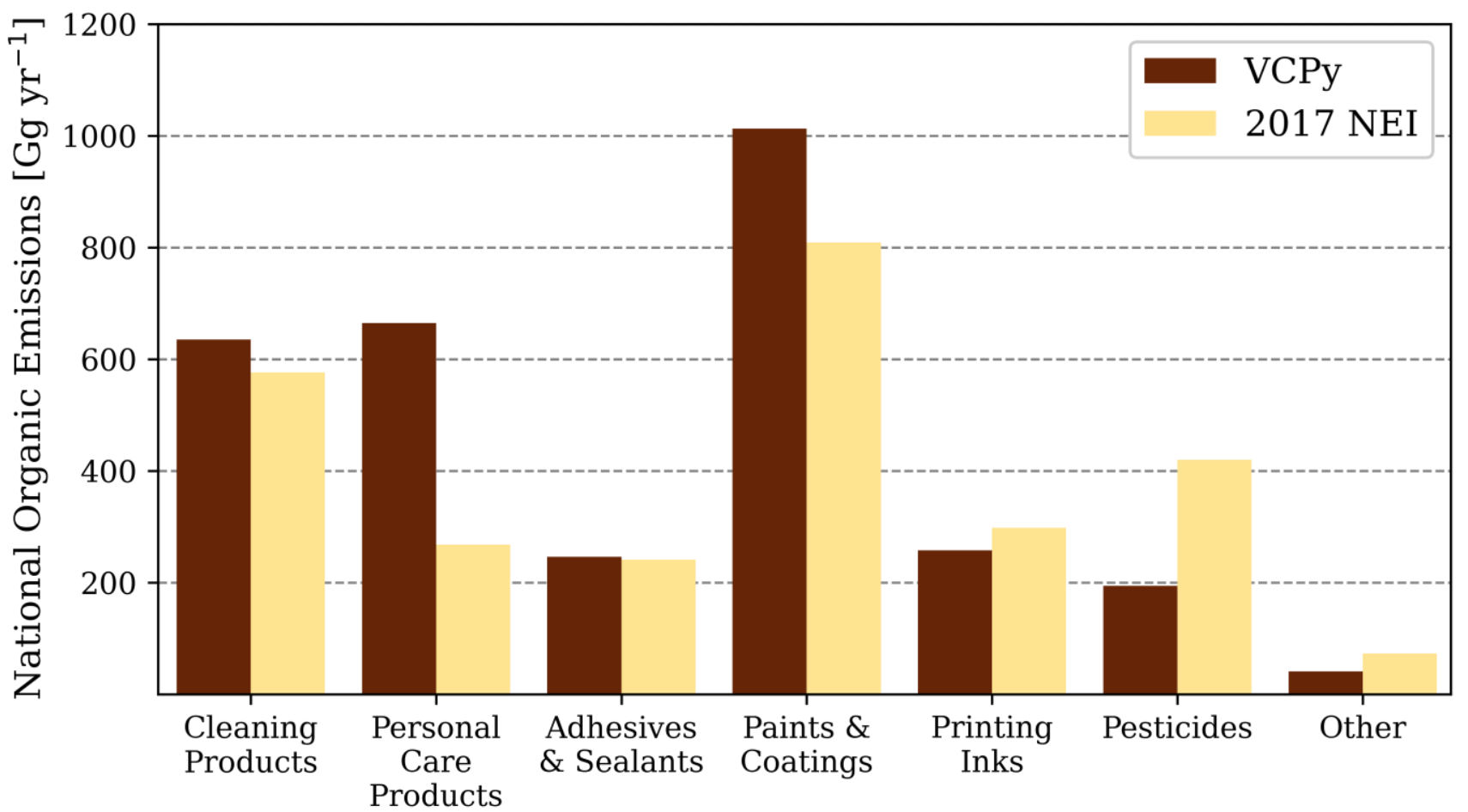

125 Figure S5: Product Use Category comparison of national-level emissions from the VCPy and 2017 NEI inventories for VCPs. For "Other," asphalt emissions in the 2017 NEI are excluded as those emissions are not quantified in the VCPy inventory. 

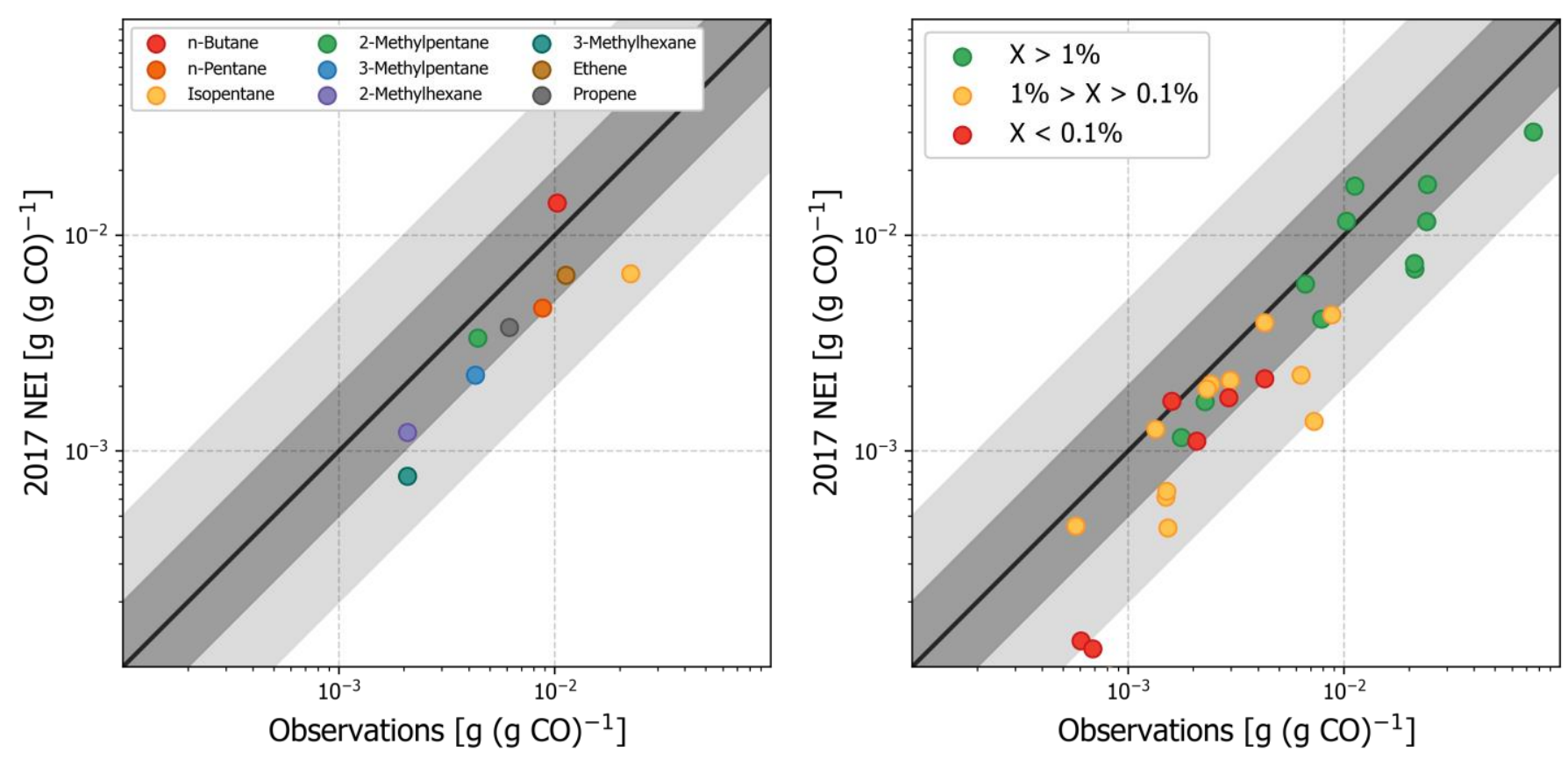

Figure S6: (Left) Evaluation of organic emission ratios of species that feature high emission factors from mobile sources in Los Angeles County using observed emission ratios from summer 2010. (Right) Evaluation of 2017 NEI organic emission ratios in Los Angeles County using observed emission ratios from summer 2010. The scatter point colors represent the relative abundance of each compound in the complete VCP sector. For example, all green points represent compounds that are $>1 \%$ of the total VCP emissions in Los Angeles County. Black line - 1:1; Dark grey shading - 2:1; Light grey shading - 5:1. 
135
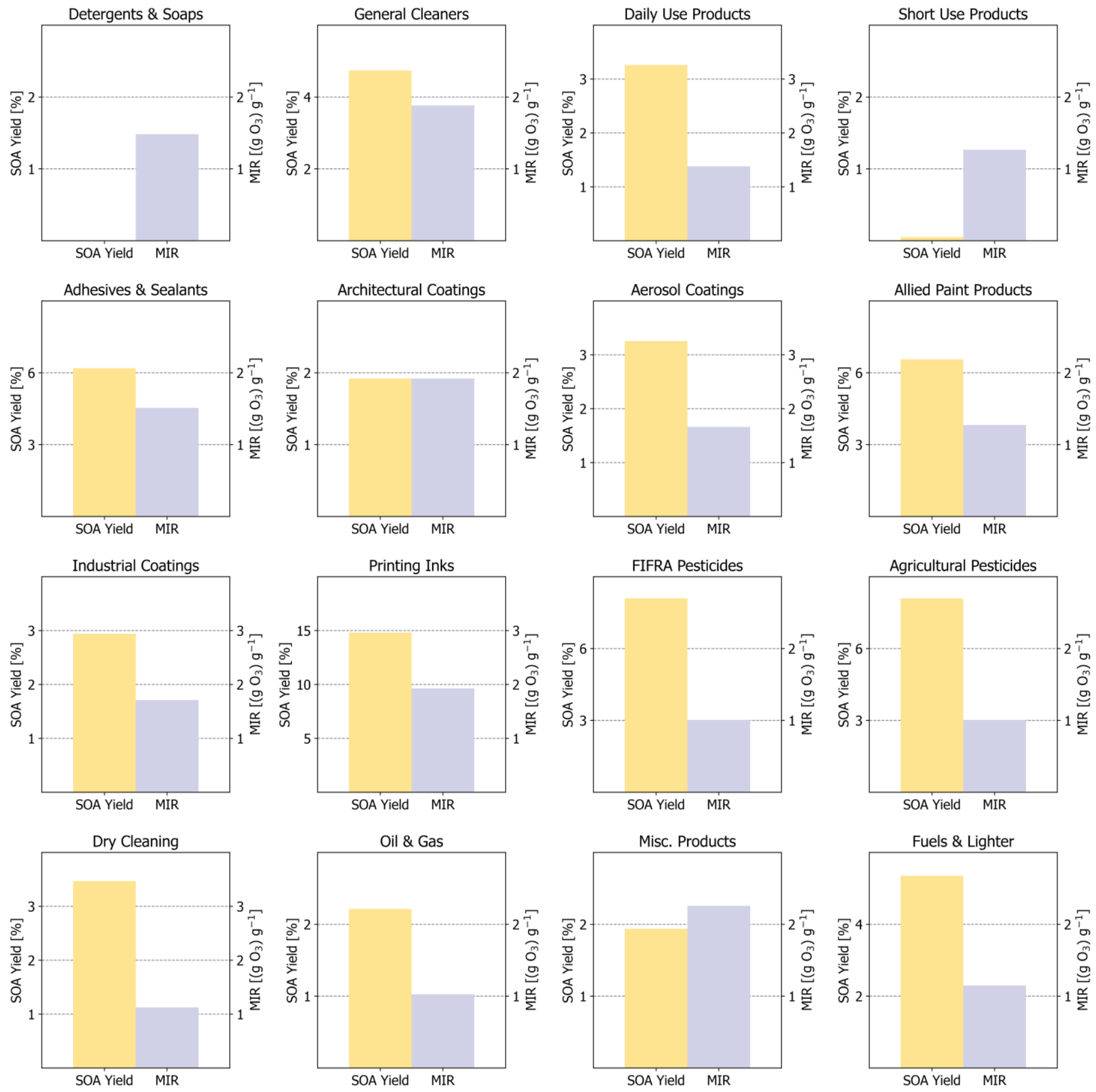

Figure S7: Effective SOA yield and MIR for all sub-PUCs. 


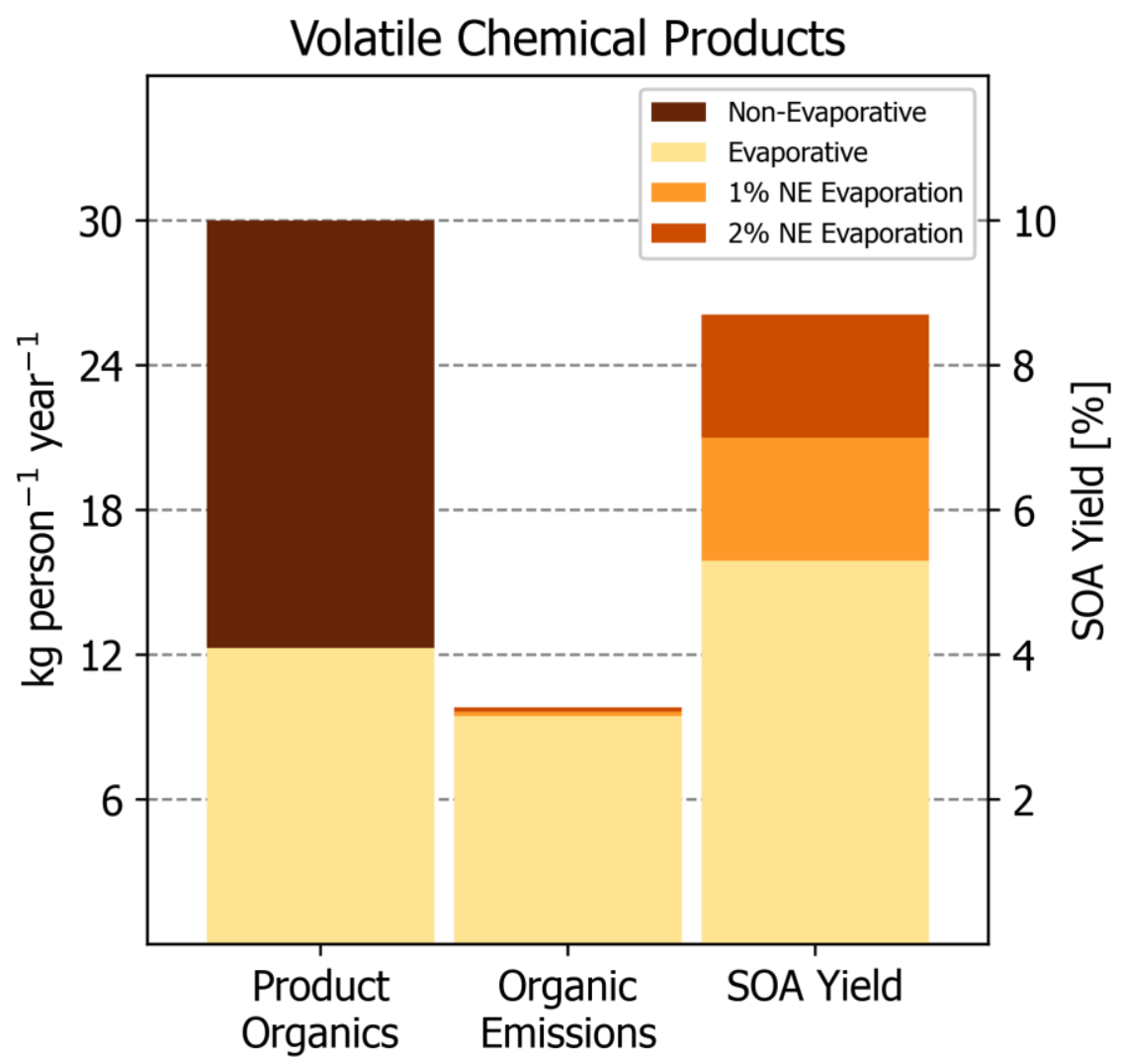

Figure S8: Total product organics, organic emissions, and sector-wide effective SOA yields resulting from adjusted non-evaporative assumptions. The two sensitivity tests are assuming $1 \%$ and $2 \%$ of all non-evaporative organic mass in VCPs evaporates and forms SOA with $100 \%$ efficiency. 